\title{
Plantas do cerrado com atividade antimicrobiana: uma revisão sistemática da literatura
}

\author{
Cerrado plants with Antimicrobial Activity: a systematic literature review
}

Plantas de cerrado con actividad antimicrobiana: una revisión sistemática de la literatura

Recebido: 03/11/2021 | Revisado: 10/11/2021 | Aceito: 23/11/2021 | Publicado: 04/12/2021

\author{
Gladyane Mendes Belém \\ ORCID: https://orcid.org/0000-0000-3360-5538 \\ Universidade Estadual de Montes Claros, Brasil \\ E-mail: gladyanebelem@gmail.com \\ Otávio Cardoso Filho \\ ORCID: https://orcid.org/0000-0001-7694-0590 \\ Universidade Estadual de Montes Claros, Brasil \\ Faculdade de Ciências Odontológicas, Brasil \\ E-mail: otaviobiol@hotmail.com \\ Francine Souza Alves da Fonseca \\ ORCID: https://orcid.org/0000-0002-5815-4550 \\ Universidade Federal de Minas Gerais, Brasil \\ E-mail: francinefonseca@yahoo.com.br \\ Eduardo Robson Duarte \\ ORCID: https://orcid.org/0000-0002-2205-9412 \\ Universidade Federal de Minas Gerais, Brasil \\ E-mail: duartevet@hotmail.com, duartevet@pq.cnpq.br
}

\begin{abstract}
Resumo
Objetivou-se descrever e relacionar compostos de plantas do Cerrado com ação inibitória para microrganismos patogênicos para humanos e animais. Para tanto, realizou-se uma busca preliminar nos Descritores em Ciências da Saúde (DeCS) para definir as palavras a serem utilizadas no levantamento da literatura. Definiu como palavras chaves, "Antimicrobiano ou Bactericida ou Fungicida" e "Planta ou Vegetal" e "Cerrado ou Bioma Cerrado". Por meio da ferramenta Medical Subject Headings (MeSH), selecionou estudos em que a combinação de palavras chaves foram identificadas nos títulos e ou resumos dos estudos. A busca pelos artigos foi realizada nas bases de dados Science Direct,Web of Science e PubMed. Foram revelados inicialmente 48 estudos, após leitura na integra foram incluídos para análise, 10 artigos publicados nos entre os anos (2017-2021). Após avaliação dos estudos, identificouse 13 espécies vegetais pertencentes a 8 famílias botânicas com potencial antimicrobiano, sendo Fabaceae a família com maior número de espécies. Para extração dos compostos, a folha das plantas foi o órgão mais usado e o etanol, como solvente. Os compostos vegetais bioativos majoritários foram terpenos e compostos fenólicos. Os metabólitos secundários detectados nos extratos das plantas apresentaram diferentes formas de ação para inibição microbiana, sob diferentes concentrações. Os compostos vegetais do Cerrado são promissores como alternativas aos antimicrobianos convencionais, sendo importantes para produção de novos fármacos. Os estudos analisados demonstraram que existem espécies vegetais nativas do Cerrado já estudadas com notório potencial antimicrobiano.
\end{abstract}

Palavras-chave: Savana; Bactericida; Fungicida; Extrato vegetal; Microdiluição; Resistência microbiana.

\begin{abstract}
The objective was to describe and relate compounds from Cerrado plants with inhibitory action against pathogenic microorganisms for humans and animals. Therefore, a preliminary search was carried out in the Health Sciences Descriptors (DeCS) to define the words to be used in the literature survey. It defined as key words, "Antimicrobial or Bactericide or Fungicide" and "Plant or Vegetal" and "Cerrado or Cerrado Biome". Through the Medical Subject Headings $(\mathrm{MeSH})$ tool, it selected studies in which the combination of keywords were identified in the titles and/or abstracts of the studies. The search for articles was performed in the Science Direct, Web of Science and PubMed databases. Initially 48 studies were revealed, after reading in full were included for analysis, 10 articles published between the years (2017-2021). After evaluating the studies, 13 plant species belonging to 8 botanical families with antimicrobial potential were identified, with Fabaceae being the family with the highest number of species. For the extraction of compounds, plant leaves were the most used organ and ethanol, as a solvent. The major bioactive plant compounds were terpenes and phenolic compounds. The secondary metabolites detected in plant extracts showed
\end{abstract}


different forms of action for microbial inhibition, under different concentrations. Plant compounds from the Cerrado are promising as alternatives to conventional antimicrobials, being important for the production of new drugs. The studies analyzed demonstrated that there are plant species native to the Cerrado that have already been studied with notorious antimicrobial potential.

Keywords: Savannah; Bactericide; Fungicide; Plant extract; Microdilution; Microbial resistance.

\section{Resumen}

El objetivo fue describir y relacionar compuestos de plantas del Cerrado con acción inhibidora de microorganismos patógenos para humanos y animales. Por ello, se realizó una búsqueda preliminar en los Descriptores de Ciencias de la Salud (DeCS) para definir las palabras a utilizar en la encuesta de literatura. Definió como palabras clave, "Antimicrobiano o Bactericida o Fungicida" y "Planta o Vegetal" y "Cerrado o Bioma Cerrado". A través de la herramienta Medical Subject Headings (MeSH), seleccionó estudios en los que la combinación de palabras clave se identificó en los títulos y / o resúmenes de los estudios. La búsqueda de artículos se realizó en las bases de datos Science Direct, Web of Science y PubMed. Inicialmente se revelaron 48 estudios, luego de la lectura completa se incluyeron para análisis, 10 artículos publicados entre los años (2017-2021). Luego de evaluar los estudios, se identificaron 13 especies de plantas pertenecientes a 8 familias botánicas con potencial antimicrobiano, siendo Fabaceae la familia con mayor número de especies. Para la extracción de compuestos, las hojas de las plantas fueron el órgano más utilizado y el etanol, como solvente. Los principales compuestos vegetales bioactivos fueron los terpenos y los compuestos fenólicos. Los metabolitos secundarios detectados en extractos de plantas mostraron diferentes formas de acción para la inhibición microbiana, a diferentes concentraciones. Los compuestos vegetales del Cerrado son prometedores como alternativas a los antimicrobianos convencionales, siendo importantes para la producción de nuevos medicamentos. Los estudios analizados demostraron que existen especies vegetales autóctonas del Cerrado que ya han sido estudiadas con notorio potencial antimicrobiano.

Palabras clave: Savannah; Bactericida; Fungicida; Extracto vegetal; Microdilución; Resistencia microbiana.

\section{Introdução}

Apesar do crescente avanço no desenvolvimento de antimicrobianos, as doenças infecciosas ainda são consideradas uma das principais causas de morbidade e mortalidade em todo o mundo, em decorrência do aumento de cepas de microrganismos patogênicos resistentes a esses fármacos. Esses fatos ocorrem devido à utilização excessiva de agentes antimicrobianos de amplo espectro, tanto em humanos como em animais (Coelho, et al., 2018).

Os microrganismos possuem a capacidade de responder de forma rápida e eficiente, às mudanças do microambiente onde estão inseridos (Monteiro, 2010). Portanto, uma pequena alteração em nucleotídeos do genoma microbiano pode resultar em resistência a agentes antimicrobianos de diferentes classes farmacêuticas (Couto et al., 2009). Dessa forma, a busca por compostos vegetais tem sido uma alternativa promissora para reduzir a ocorrência de cepas resistentes aos antimicrobianos convencionais (Brandão et al., 2009).

O Brasil é detentor de uma flora diversificada e extensa, com aproximadamente 40.000 mil espécies vegetais com grande potencial para o desenvolvimento de conhecimento e produtos (Carvalho \&Cante, 2021). O Cerrado, um dos principais biomas do Brasil, devido à grande diversidade de sua flora apresenta números crescentes de estudos relacionados aos componentes naturais das plantas ricos em propriedades bioativas (Patridge et al., 2016). Os terpenos, compostos fenólicos e alcaloides têm demonstrado alta capacidade de inibir o crescimento microbiano, o que tem favorecido avanço da indústria farmacêutica para desenvolvimento de novos antimicrobianos (Srivastav et al., 2019; Prakash et al., 2020).

Entretanto, faz-se necessário conhecer as principais espécies vegetais do Cerrado estudadas, bem como seus efeitos na inibição microbiológica. Neste estudo, objetivou-se descrever e relacionar compostos de plantas do Cerrado com ação inibitória para microrganismos patogênicos para humanos e animais.

\section{Metodologia}

O presente estudo trata-se de uma revisão sistemática da literatura que investigou abordagens farmacológicas de espécies vegetais do Cerrado com propriedades antimicrobianas. As principais etapas foram conduzidas de acordo com a 
metodologia estabelecida pelos itens Preferred Reporting Items is Systematic Reviews and Metanalyses (Prisma) (Galvão et al., 2015).

$\mathrm{O}$ estudo incluiu artigos originais que investigaram abordagens farmacológicas de extratos vegetais no controle microbiano de publicações nos últimos cinco anos (2017-2021) escritos em língua inglesa e portuguesa. As bases de dados consultadas e analisadas para seleção dos artigos foram: Science Direct, Web of Science, e PubMed.

Realizou-se uma busca preliminar nos Descritores em Ciências da Saúde (DeCS) (http://decs.bvs.br/) para identificar os termos a serem utilizados no levantamento da literatura. Por meio da ferramenta Medical SubjectHeadings (MeSH), realizou a combinação de palavras chaves e em seguida, foram selecionados estudos em que as palavras chaves foram identificadas nos títulos e ou resumos dos estudos. Os termos/palavras chaves usados foram: Antimicrobiano OU Bactericida OU fungicida OU antibacteriano OU antifúngico, E planta OR vegetal E Cerrado OR Bioma Cerrado. O objetivo deste procedimento não foi apenas filtrar os resultados, mas também cruzar os termos principais para obter o máximo de estudos possíveis. Os autores revisores deste estudo tentaram identificar todos os termos e seus sinônimos nos títulos ou resumos dos artigos para posterior leitura do artigo completo na íntegra.

Dois revisores Cardoso-Filho, O. e Duarte, E. R. realizaram a primeira triagem dos títulos e resumos para selecionar os estudos elegíveis e, em seguida, avaliaram os registros de forma independente (Sandelowski \& Barroso, 2007). Para tanto foram seguidas três etapas: 1) leitura do título; 2) leitura do resumo; 3) leitura do texto completo. Na primeira etapa, foram selecionados os estudos que apresentassem a combinação de pelo menos dois termos (ou sinônimos) identificados no MeSH. $\mathrm{Na}$ segunda etapa, foram selecionados apenas artigos com dados suficientes sobre a intervenção realizada (abordagem farmacológica antimicrobiana) e desfechos analisados (efeito antimicrobiano do composto vegetal). Na etapa final, durante a leitura do texto integral, foram considerados os principais resultados. Foram excluídos, estudos que não avaliaram o efeito do extrato; estudos que avaliaram o efeito do composto com dados secundários; livros, teses dissertações, artigos de revisão e estudos que não citaram a espécie vegetal estudada.

As espécies botânicas citadas nos estudos avaliados foram confirmadas, identificadas e listadas nos resultados segundo o site Flora Brasil 2020 (http://floradobrasil.jbrj.gov.br/reflora/listaBrasil/UC.do\#CondicaoTaxonCP).

\section{Resultados e Discussão}

Um fluxograma mostrando a quantidade de artigos identificados, excluídos e incluídos em cada etapa está apresentado na Figura 1. 
Figura 1: Organograma da seleção dos artigos de interesse.

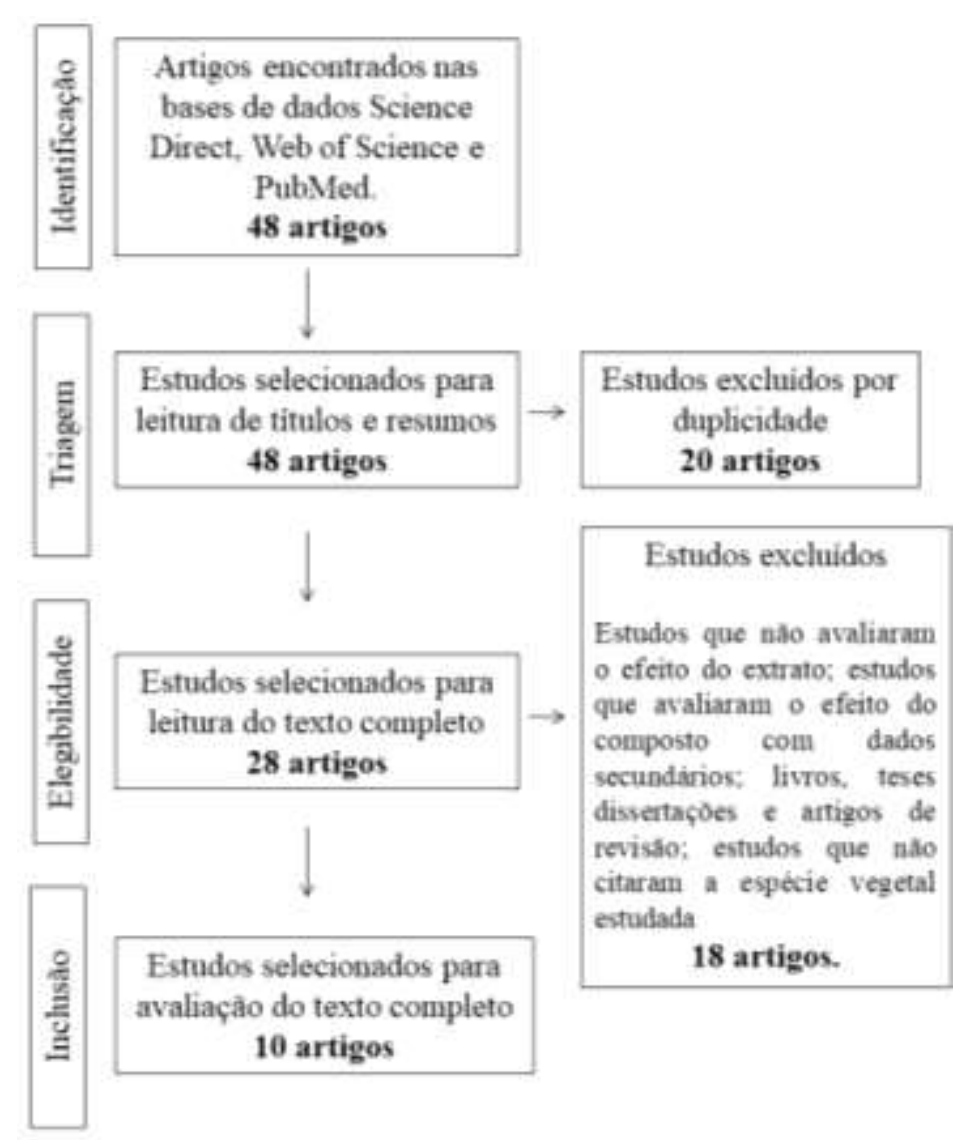

Fonte: Autores.

Inicialmente, nas pesquisas de base de dados foi identificado um total de 42 artigos com potencial para integrar a revisão, após a leitura dos títulos e resumos 28 artigos foram analisados na íntegra, sendo selecionados, 10 estudos para avaliação. As espécies botânicas selecionadas foram categorizadas da seguinte forma; família, nome botânico, parte da planta coletada, solventes de extração, microrganismos inibidos, compostos bioativos com ação antimicrobiana e suas respectivas referências.

Foi possível identificar dez publicações em artigos científicos que reportaram e a atividade antimicrobiana de plantas do Cerrado. Esses estudos indicaram treze espécies pertencentes às oito famílias botânicas, sendo Myrtaceae, a família com maior número de espécies vegetais com efeito antimicrobiano, seguido da família Arecaceae (Tabela1). 
Tabela 1: Famílias botânicas, espécies vegetais e nomes comuns de plantas do cerrado com efeito antimicrobiano citadas nos estudos selecionados.

\begin{tabular}{|c|c|c|}
\hline Família vegetal & Espécie vegetal & Nome Comum do vegetal \\
\hline Arecaceae & $\begin{array}{l}\text { Mauritia flexuosa L.f } \\
\text { Orbignya speciosa (Mart.) Barb.Rodr. }\end{array}$ & $\begin{array}{l}\text { Buriti } \\
\text { Babaçu }\end{array}$ \\
\hline Bixaceae & Cocholospermumregium (Schrank) Pilg. & Agodão-do-cerrado \\
\hline Caryocaceae & Cariocar cariaceum Wittm. & Pequi \\
\hline Euphorbiaceae & $\begin{array}{l}\text { Croton lundianus (Didr.) Müll.Arg. } \\
\text { Croton betaceus Baill. }\end{array}$ & $\begin{array}{l}\text { Velame Amarelo } \\
\text { Velame }\end{array}$ \\
\hline Fabaceae & Inga laurina (Sw.) Willd. & Ingá \\
\hline Myrtaceae & $\begin{array}{l}\text { Campomanesia adamatium (Cambess.) O. Berg } \\
\text { Eugenia klotzschiana O. Berg } \\
\text { Myrcia bela Cambess. } \\
\text { Myrcia fallax Rich.) DC. }\end{array}$ & $\begin{array}{l}\text { Guabiroba } \\
\text { Pêra-do-cerrado } \\
\text { Mircia } \\
\text { Mircia }\end{array}$ \\
\hline $\begin{array}{l}\text { Simaroubaceae } \\
\text { Sterculiaceae }\end{array}$ & $\begin{array}{l}\text { Simabaferruginea A. St.-Hil. } \\
\text { Sterculiastriata A. St.-Hil. \& Naudin }\end{array}$ & $\begin{array}{l}\text { Calunga } \\
\text { Chicha }\end{array}$ \\
\hline
\end{tabular}

Fonte: Autores.

Constatou-se ainda que $60 \%$ dos estudos utilizaram o etanol e 50\% utilizaram a água como solventes para extração dos compostos vegetais. A folha das plantas foi o órgão mais usado para obtenção dos extratos, $60 \%$ dos estudos, seguido do fruto, $30 \%$. Os estudos atribuíram a atividade antimicrobiana das espécies analisadas aos compostos fenólicos, identificados em sete estudos e aos terpenos, identificados em quatro estudos, sendo essas classes de compostos presentes em diferentes famílias botânicas. A tabela 2 relaciona as espécies de plantas do Cerrado à parte utilizada para o extrato mostraram diferentes doses para obtenção do efeito antimicrobiano em função do composto presente no extrato e do microrganismo alvo (Tabela 2). 


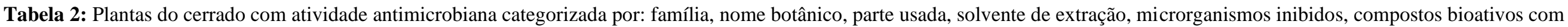
ação antimicrobiana e referências.

\begin{tabular}{|c|c|c|c|c|c|c|}
\hline Autor/Ano & Espécie Botânica & $\begin{array}{l}\text { Parte da } \\
\text { Planta }\end{array}$ & $\begin{array}{l}\text { Solvente de } \\
\text { extração }\end{array}$ & $\begin{array}{l}\text { Compostos } \\
\text { Bioativos }\end{array}$ & $\begin{array}{l}\text { Microrganismos } \\
\text { Inibidos }\end{array}$ & $\begin{array}{l}\text { Concentração } \\
\text { com eficácia }\end{array}$ \\
\hline Alves et al.,2017 & Cocholospermum regium & $\begin{array}{l}\text { Casca e poupa } \\
\text { do fruto }\end{array}$ & Etanol & $\begin{array}{l}\text { Compostos } \\
\text { fenólicos e } \\
\text { terpenos }\end{array}$ & $\begin{array}{c}\text { Malassezia spp. } \\
\text { Microsporum canis E. Bodin ex Guég. } 1902\end{array}$ & $\begin{array}{l}2,44 \mu \mathrm{g} / \mathrm{mL} \\
2,44 \mu \mathrm{g} / \mathrm{mL}\end{array}$ \\
\hline Carneiro et al., 2017 & Eugenia klotzschiana & $\begin{array}{l}\text { Folha } \\
\text { Flor }\end{array}$ & Água & Terpenos & $\begin{array}{l}\text { Streptococcus salivarius } \\
\text { Streptococcus mutans } \\
\text { Streptococcus mitis } \\
\text { Prevotella nigrescens }\end{array}$ & $\begin{array}{l}200 \mu \mathrm{g} / \mathrm{mL} \\
50 \mu \mathrm{g} / \mathrm{mL} \\
200 \mu \mathrm{g} / \mathrm{mL} \\
50 \mu \mathrm{g} / \mathrm{mL}\end{array}$ \\
\hline Santos et al., 2018 & $\begin{array}{l}\text { Myrcia bela } \\
\text { Myrcia fallax }\end{array}$ & $\begin{array}{l}\text { Folha } \\
\text { Folha }\end{array}$ & $\begin{array}{l}\text { Hidroetanol } \\
\text { Hidroetanol }\end{array}$ & $\begin{array}{l}\text { Compostos } \\
\text { fenólicos }\end{array}$ & $\begin{array}{l}\text { E coli } \mathrm{EPEC} \\
\text { E. coli } \mathrm{EPEC}\end{array}$ & $\begin{array}{l}300 \mu \mathrm{g} / \mathrm{mL} \\
250 \mu \mathrm{g} / \mathrm{mL}\end{array}$ \\
\hline
\end{tabular}


Continuação

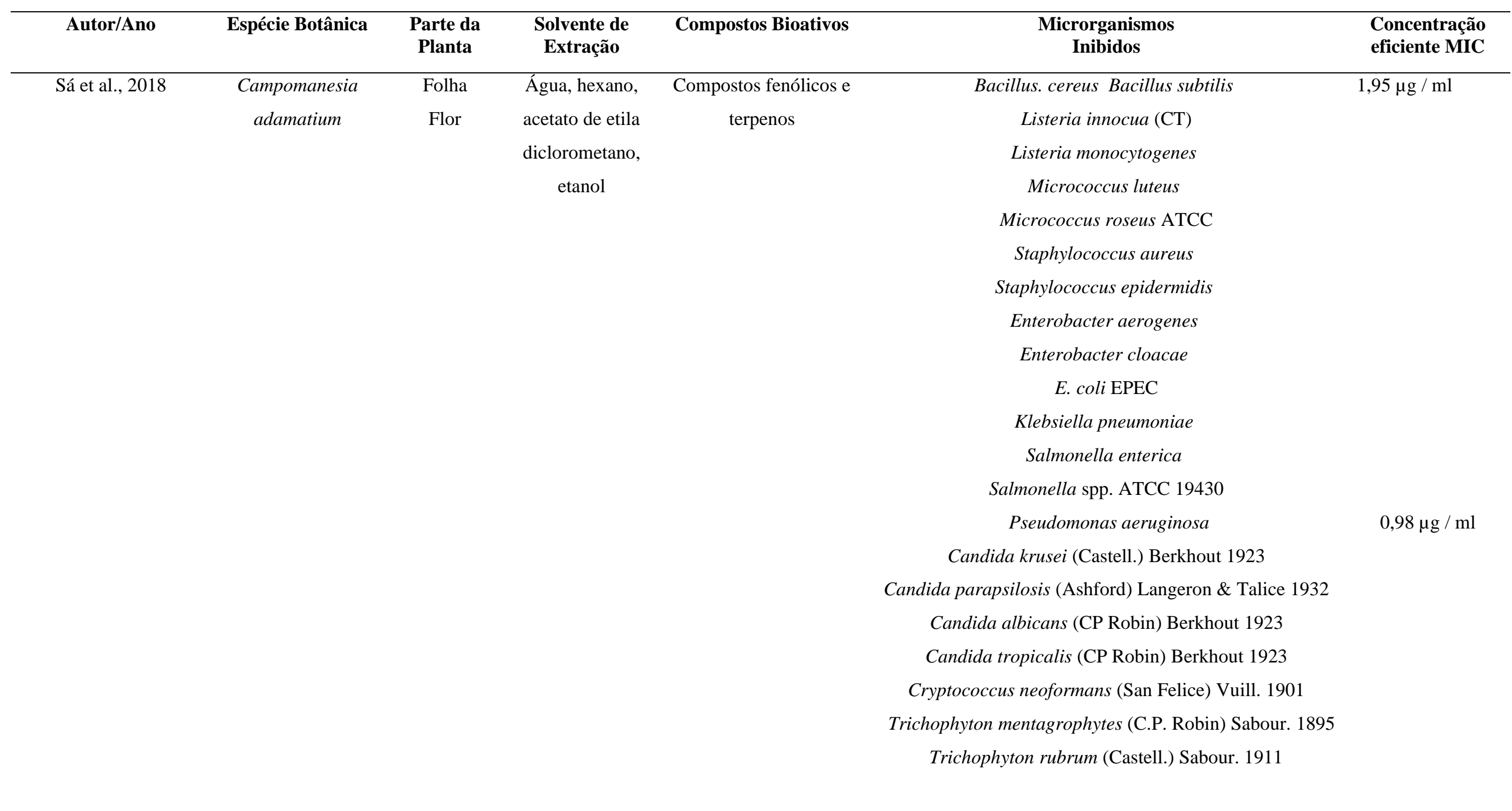




\begin{tabular}{|c|c|c|c|c|c|c|}
\hline & & & & & & Continuação \\
\hline Autor/Ano & Espécie Botânica & $\begin{array}{l}\text { Parte da } \\
\text { Planta }\end{array}$ & $\begin{array}{l}\text { Solvente de } \\
\text { Extração }\end{array}$ & $\begin{array}{l}\text { Compostos } \\
\text { Bioativos }\end{array}$ & $\begin{array}{l}\text { Microrganismos } \\
\text { Inibidos }\end{array}$ & $\begin{array}{l}\text { Concentração } \\
\text { eficiente MIC }\end{array}$ \\
\hline \multirow[t]{3}{*}{ Nobre et al., 2018} & Mauritia flexuosa & Fruto & Hexano & Ácido graxo & E. coli & $32 \mu \mathrm{g} / \mathrm{mL}$ \\
\hline & & & & & S. aureus & $256 \mu \mathrm{g} / \mathrm{mL}$ \\
\hline & Orbignya speciosa & Fruto & Hexano & Ácido graxo & E. coli & $512 \mu \mathrm{g} / \mathrm{mL}$ \\
\hline \multirow[t]{4}{*}{ Gonzani et al., 2018.} & Simaba ferruginea & Rizoma & Metanol & Alcaloide & S. aureus & $12,5 \mu \mathrm{g} / \mathrm{mL}$ \\
\hline & & & & Compostos fenólicos. & E. coli & $25 \mu \mathrm{g} / \mathrm{mL}$ \\
\hline & & & & & E. faecalis & $200 \mu \mathrm{g} / \mathrm{mL}$ \\
\hline & & & & & Shigella flexneri & $200 \mu \mathrm{g} / \mathrm{mL}$ \\
\hline Martins et al.,2019 & Ingalaurina & Folha & $\begin{array}{l}\text { Etanol } \\
\text { Água }\end{array}$ & Compostos fenólicos & Candida glabrata (H.W.Anderson) S.A. Mey. \& Yarrow 1978 & $11,7 \mu \mathrm{g} / \mathrm{mL}$ \\
\hline Silva et al., 2020 & Sterculia striata & $\begin{array}{l}\text { Exsudato da } \\
\text { casca }\end{array}$ & $\begin{array}{l}\text { Acetona } \\
\text { Etanol }\end{array}$ & Heteropolissacarídeos & S.aureus . & $100 \mu \mathrm{g} / \mathrm{mL}$ \\
\hline Rocha et al., 2021 & Croton betaceus & Folha & $\begin{array}{l}\text { Acetato de etila, } \\
\text { água, etanol, } \\
\text { metanol, hexano, } \\
\text { clorofórmio }\end{array}$ & $\begin{array}{c}\text { Compostos fenólicos } \\
\text { Terpenos }\end{array}$ & E. faecalis & $100 \mu \mathrm{g} / \mathrm{mL}$ \\
\hline
\end{tabular}

Fonte: Autores. 
Constou-se que $80 \%$ das pesquisas indicaram efeitos antibacterianos (incluindo bactérias consideradas Grampositivas e Gram-negativas) e 46\% delas descreveram efeitos antifúngicos. Quanto aos mecanismos de ação dos compostos, foi relatado nos trabalhos avaliado que a ação detergente e/ou aspectos hidrofóbicos estejam relacionados à entrada de compostos pelas membranas dos microrganismos, causando danos na sua estrutura e garantindo o efeito do extrato vegetal sobre o microrganismo (Figura 2).

Figura 2: Representação geral dos achados após avaliação dos estudos contemplados na revisão. Principal família vegetal do Cerrado, solventes utilizados para obtenção dos extratos, compostos mais importantes presentes nos extratos e possível mecanismo de ação dos compostos sobre os microrganismos.

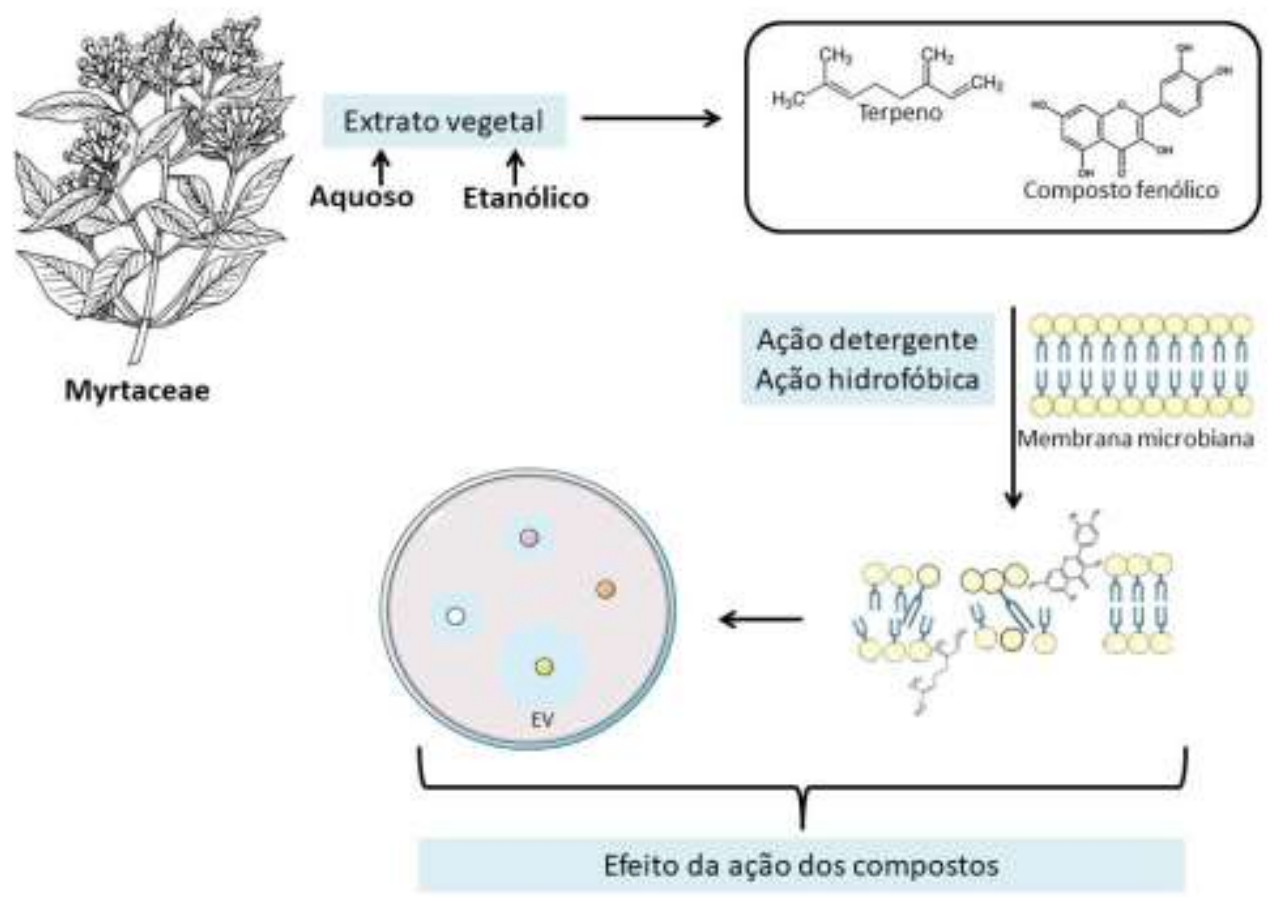

Fonte: Autoria própria a partir de figuras disponíveis em https://smart.servier.com/

O Bioma Cerrado é considerado um hotspot global, pois apresenta diversidade de espécies vegetais nativas que correspondem, aproximadamente, a 5\% da diversidade mundial (Brasil, 2010). Esse Bioma possui inúmeras espécies de plantas com propriedades medicinais, por isso a importância da realização de pesquisas para elucidar melhor essa potencialidade (Oliveira; Viveiro, 2012).

A família Myrtaceae compreende aproximadamente 5.000 espécies e 144 gêneros no mundo. No Brasil a família abrange 23 gêneros e cerca de 1.000 espécies (Sobral et al. 2015). Os frutos e folhas dessa família são amplamente utilizados na medicina popular e, por isso, muitos estudos já foram realizados a fim de elucidar suas aplicações medicinais. A atividade consagrada na cultura popular e também em pesquisas científicas fez com que espécies desta família fizessem parte da World Health Organization em 2002.

Entre os gêneros de Myrtaceae, Campomanesia, Eugenia e Myrcia são descritos na literatura como plantas com efeito antibacteriano e antifúngico (Dexeimer \& Pozzobon, 2017). O gênero Campomanesia demonstrou eficiência na atividade antifúngica para a fração aquosa e antibacteriana na fração hexânica (Cardoso et al., 2010, Sá et al., 2018), outros estudos, relataram a atividade antimicrobiana desse genêro (Bonilla et al, 2005; Pavan, et al. 2009; Brandelli et al. 2013). Metabólitos secundários encontrados no gênero Eugenia por (Limberger et al., 1998, Medeiros et al., 2003, Stefanello et al., 
2008, Simonetti et al., 2016, Kauffmann et al., 2017) e no gênero Myrcia por Alarón et al. (2009) mostraram potencial antibacteriano e antifúngica por meio de avaliação do extrato etanólico em diferentes concentrações. Corroborando com resultados de outros estudos que também mostraram ação antimicrobiana do extrato metanólico dos gêneros Eugenia e Myrcia (Cascaes et al., 2021).

Todas as pesquisas disponíveis na literatura mostram diversidade nas concentrações mínimas para efeito do composto sobre a atividade antimicrobiana. Isso provavelmente está associado à estrutura morfológica do microrganismo, o solvente utilizado para obtenção do extrato e os grupos de compostos identificados. Essa informação reforça o achado apresentado por Tavares et al. (2020), cujo estudo mostrou que bactérias gram-positivas exigem concentrações maiores que as Gram-negativas, para que ocorra a atividade antimicrobiana. Por outro lado, estudo utilizando outra classe de compostos (fenólicos) e uma cepa bacteriana diferente, revelou maior sensibilidade ao composto em bactérias gram-positivas (BouarabChibane et al., 2019), o que estabelece a necessidade de novos estudos para melhor compreensão sobre o mecanismo de ação dos compostos na promoção do controle microbiano.

Ainda sobre o possível mecanismo de ação mostrado na figura 02, alguns autores relatam informações que fortalece a possibilidade de uma associação ao mecanismo de entrada do composto no organismo alvo. Segundo Nobre et al. (2018), as biomoléculas que apresentam capacidade de efeito antimicrobiano, interrompem a integridade da membrana celular e facilita a liberação de constituintes intracelulares vitais e inibem enzimas alvo (Oz et al. 2015). Podem ainda modificar o pH da célula e causar interferência no sistema de geração de ATP (Djilani \& Dicko, 2012). Sendo essas as possíveis causas do dano microbiano. Segundo_Bouarab-Chibane et al. (2019) os efeitos desses compostos são heterogêneos, por isso sua ação no estimulo do crescimento bacteriano e atividade antibacteriana provavelmente dependem da cepa bacteriana.

No que diz respeito aos compostos com efeito positivo no controle de fungos e bactérias, os resultados encontrados nessa revisão apontaram fenólicos e terpenos como os mais importantes e esses por sua vez, corroboram com os resultados de outros estudos. Os fenólicos apresentam potencial antimicrobiano contra um amplo espectro de microrganismos e possuem capacidade de tornar cepas resistentes a antibióticos sensíveis ao antimicrobiano natural (Quideau, et al., 2011, Miklasinska et al., 2016, Zacchinho et al., 2017). Os terpenos são compostos conhecidos por suas propriedades antimicrobianas por seus efeitos prejudiciais na estrutura e função das membranas microbianas e paredes celulares Andrade-Ochoa et. al. (2015), resultado de suas propriedades lipofílicas, da potência de seus grupos funcionais e de sua solubilidade em água (Dorman \& Deans, 2000).

Devido à relevância desses compostos bioativos vale ressaltar aqui suas características químicas, tendo em vista a contribuição para possibilidades de inovação na farmacologia, como estratégia para obtenção de fármacos com potencial para controle de microrganismos causadores de doenças animais e humanas. Os compostos fenólicos são metabólitos secundários vegetais que derivam em classes, flavonóides e não flavonoides, derivados hidroxilados de anéis benzeno e amplamente distribuídos nas plantas, além funcionar naturalmente como defesa em espécies vegetais (Boudet, 2007). Já os terpenos, é um dos grupos mais complexos de metabólitos secundários encontrados nas plantas, são os principais constituintes dos óleos essenciais, derivados da via dos isoprenóides e são produzidos e segregados de tecidos vegetais especializados (Iriti et al., 2006)

\section{Considerações Finais}

Os estudos analisados demonstraram que existem espécies vegetais nativas do Cerrado já estudadas com notório potencial antimicrobiano, com destaque naquelas pertencentes às famílias botânicas Myrtaceae e Arecaceae. E revelou ainda que essas plantas apresentam compostos como fenólicos e terpenos com potencial para a utilização de formas isoladas ou em associação, como matéria prima para produção de novos fármacos com ação inibidora frente a patógenos microbiológicos de 
importância humana e animal. Diante disso, torna-se imprescindível a adoção de estratégias que favoreçam a conservação do Bioma Cerrado.

Sugere ainda, que sejam realizados novos estudos com diferentes famílias botânicas, a fim de conhecer novas espécies vegetais com propriedades medicinais e estudos aprofundados para revelar o mecanismo de ação dos compostos sobre os organismos alvos.

\section{Referências}

Alarcón LD, Peña AE, Quintero A, Meza M, Usubillaga A, Velasco J. (2009). Composición y actividad antibacteriana del aceite esencial de Myrcia fallax (Rich.) Dc de Venezuela. Revista de la Sociedad Química del Perú, 75 (2), 221-227.

Andrade, L. B. S., Julião, M. S. S., Vera Cruz, R. C., Rodrigues, T. H. S., Fontenelle, R. O. S., \& Silva, A. L. C. (2018). Antioxidant and antifungal activity of carnauba wax powder extracts, Industrial Crops and Products, 12 (5), 220-227.

Andrade-Ochoa S., Nevárez-Moorillón G.V, Sánchez-Torres L.E. (2015). Relação quantitativa estrutura-atividade de moléculas constituintes de diferentes óleos essenciais com atividade antimicobacteriana contra Mycobacterium tuberculosis e Mycobacterium bovis. BMC Complem Altern Med, 1 (5), 333-342.

Bonilla, A., Duque, C., Garzón, C., Takaishi, Y., Yamaguchi, K., Hara, N., Fujimoto, Y., (2005). Champanones, yellow pigments from the seeds of champa (Campomanesia lineatifolia), Phytochemistry, 66 (14), 1736-1740.

Bouarab-Chibane, L., Forquet. V., Lantéri, P., Clément,Y., Léonard-Akkari, L., Oulahal, N., Degraeve, P. E., Bordes, C. 2019. Antibacterial Properties Of Polyphenols: Characterization And Qsar (Quantitative Structure-Activity Relationship) Models. Front. Microbiol, 9 (9), $1457-1474$.

Boudet A. (2007). Evolução e estado atual da pesquisa em compostos fenólicos. Fitoquímica. Jornal phytochem. 68 (2), $2722-2735$. doi: 10.1016 /.06.012.

Brandão, M. GL et al. (2009). Traditional uses of American plant species from the 1st edition of Brazilian Official Pharmacopoeia. Revista Brasileira de Farmacognosia, 19 (2), 478-487.

Brandelli, C. L. C., Vieira, P. de B., Macedo, A. J., Tasca, T. (2013). Remarkable Anti-Trichomonas vaginalis activity of plants traditionally used by the MbyáGuarani indigenous group in Brazil. BioMed Research International, 20 (13), 1-7. https://doi.org/10.1155/2013/826370.

Brasil, 2010. Ministério do Meio Ambiente. Plano de Ação para Prevenção e Controle do Desmatamento e das Queimadas. 2010. http://www.mma.gov.br/estruturas/201/_arquivos/ppcerrado_201.pdf accessed 14.11.18.

Campos, D. A., Ribeiro, A. C., Costa, E. M., Fernandes, J. C., Tavaria, F. K., Araruna, F. B., Eiras, C., Eaton, P., Leite, J. R. S. A., \& Manuela Pintado, M. (2012). Study of antimicrobial activi tyandatomic force microscopy imaging of the action mechanism of cashewtreegum. Carbohydr. Polym., 90 (5), 270-274.

Cardoso C. A L., Salmazzo G. R., Honda N. K., Prates C. B., Vieira M. C., and Roberta Gomes Coelho. (2010). Antimicrobial Activity of the Extracts and Fractions of Hexanic Fruits of Campomanesia Species (Myrtaceae). Journal Of Medicinal Food J Med Food, 13 (5), $1273-1276$.

Carneiro, N. S. et al. (2017). Chemical composition, antioxidant and antibacterial activities of essential oils from leaves and flowers of Eugenia klotzschiana Berg (Myrtaceae). Anais da Academia Brasileira de Ciências, 89 (03), 1907-1915.

Carvalho, A. P. A., \& Conte-Junior, C. A. (2021). Health benefitsofphytochemicalsfromBraziliannativefoodsandplants: Antioxidant, antimicrobial, anticancer, andriskfactorsofmetabolic/endocrinedisorderscontrol, Trends in Food Science \& Technology, 11(1), 534-548.

Cascaes, M. C., Guilhon, G. M., Zoghbi, M. G., Andrade, E. H. A., Santos, L. S., Silva, J. K. R., Uetanabaro A. P.T., Araújo I. S. (2021). Flavonoids, antioxidant potential and antimicrobial activity of Myrcia rufipila mcvaugh leaves (myrtaceae). Nat Prod Res., 35 (10), 1717-1721.

Couto R.C., Pedrosa T.M.G., Nogueira J.M. (2009). Infecção hospitalar e outras complicações não-infecciosas da doença - epidemiologia, controle e tratamento. Rio de Janeiro, Brasil: Guanabara Koogan.

Cowan M. (1999). Produtos vegetais como agentes antimicrobianos. Clin Microbiol Rev., 12 (2), 564-582.

Dexheimer, G. M., Pozzobon, A. (2017). Atividade biológica de plantas da família Myrtaceae: revisão sistemática de artigos entre 1989 e 2015. Revista Cubana de Plantas Medicinales, 22 (2), 321-327.

Dias M, Monteiro MS. (2010). Antibióticos e Resistência bacteriana, Velhas Questões Novo Desafio. Cadernos Otorrinolaringologia, 21 (9), 1-10.

Dorman H.J.D., Deans S.G. (2000). Agentes antimicrobianos de plantas: atividade antibacteriana de óleos voláteis de plantas. $J$ Appl Microbiol, 88 (2), 308316.

Durigan, G., Melo, A. C. G., Max, J. C. M., Vilas Boas, O., Contieri, W. A.; Ramos, V. S. (2011). Manual para recuperação da vegetação de Cerrado. São Paulo, Brasil: SMA.

Fischbach, M.A.; Walsh, C.T. (2009). Antibiotics For Emergingpathogens. Science, 32 (5), 1089-1093.

Galvão, T. F., Andrade, T. S., Harrad, P. D. (2015). Principais itens para relatar Revisões sistemáticas e Meta-análises: A recomendação PRISMA. Epidemiol. Serv. Saúde, 24 (2), 335-342. 
Gazoni, V. F., Balogun, S. O., Arunachalam, K., Oliveira, D. M., Filho, V, C., Lima, S. R., Colodel, E. Soares, M., I. M, Ascêncio, S. D., \& Martins, D. T. O. (2018). Assessment of toxicity and differential antimicrobial activity of methanol extract of rhizome of Simaba ferruginea A. St.-Hil.and its isolate canthin-6one, Journal of Ethnopharmacology, 22(3), 122-134.

Iriti M., Colnaghi G., Chemat F., Smadja J., Faoro F., Visinoni F. A. (2006). Histo-citoquímica e microscopia eletrônica de varredura de tricomas glandulares de lavanda após hidrodestilação convencional e assistida por microondas de óleos essenciais: Um estudo comparativo. Flavor Fragr. J., 21 (3), 704-712.

Kauffmann, C., Soares, A. P. V., Arossi, K., Pacheco, L. A., Buhl, B., Freitas, E. M., Hoehne, L., Castro, L. C., Gnoatto, S. C. B., Ethur, E. M. (2017). Potencial Antimicrobiano e Antibiofilme in Vitro de Espécies do Gênero Eugenia, Myrtaceae, Nativas do Sul do Brasil. Caderno pedagógico, 14 (2), $110-127$.

Lc B. B. C. , P. Marcelino, S. S., W. Felix, O., Mc Moura, E. Viana, P., F. Soares, G., P Guedes, P., Th Napoleão, M, S. C. (2018). Lectinas Como Agentes Antimicrobianos. Journal Of Aplied Microbiology, 22 (3), 238-1252.

Limberger RP, Apel MA, Sobral M, Schapoval ES, Henriques AT. (1998) Investigação da atividade antimicrobiana do óleo volátil de espécies da família Myrtaceae. Revista Brasileira de Farmácia, 79 (2), 49-52.

Loureiro, R. J., et al. (2016). O uso de antibióticos e as resistências bacterianas: brevesnotassobre a suaevolução. Revista Portuguesa de saúde pública, 34 (1), $77-84$.

Kępa M., Wojtyczka R.D., Idzik D., Dziedzic A., Wąsik T.J. (2016). Catechin hydrate aumenta a ação antibacteriana de antibióticos selecionados contra cepas clínicas de Staphylococcus aureus . Moléculas, $21: 244$

Maclean, R. C., \& San Millan, A. (2019) The evolution of antibiotic resistance. Science, 365 (6458), 1082-1083.

Mark, S. B., \& Antony D. (2006). Buss. Natural products . The future scaffolds for novel antibiotics? Biochemical Pharmacology, 71(7), 919-929.

Medeiros JR, Medeiros N, Medeiros H, Davin LB, Lewis NG. (2003). Composition of the bioactive essential oils from the leaves of Eugenia stipitata McVaugh ssp. sororia from the Azores. Journal of Essential Oil Research, 15 (4), 293-5.

Mulyaningsih , S., Sporer, F., Zimmermann, S., Reichling, J., \& Wink, M. (2010). Synergisticpropertiesoftheterpenoidsaromadendreneand 1,8-cineole fromtheessentialoilofEucalyptusglobulusagainstantibiotic-susceptibleandantibiotic-resistantpathogens. Phytomedicine, 17 (13), $1061-1066$.

Nobre, C. B., Sousa, E. O., Silva, J. M. F.L., Coutinho, H. D. M., \& Costa, J. G. M. (2018). Chemical composition and antibacterial activity of fixed oils of Mauritiaflexuosa and Orbignyaspeciosa associated with aminoglycosides, European Journal of Integrative Medicine, 23 (1), $84-89$.

Oliveira, V. B. et al. (2012). Native foods from Brazilian biodiversity as a source of bioactive compounds. Food Research International, 48 (1), $170-179$.

Organização Mundial De Saúde. (2019). Antibióticos: resistência de microrganismos é grave ameaça à saúde global.htps://portal.fiocruz.br/noticia/antibioticos-resistencia-de-microrganismos-e-grave-ameaca-saude-global

Oz M., Lozon Y., Sultan A., Yang KHS, Galadari S. (2015). Effects of monoterpenes on ion channels of excititable cells. Pharmacol. Ther, 15 (2), 83-97.

Patridge E, Gareiss P, Kinch MS , Hoyer D . (2016). Uma análise de medicamentos aprovados pela FDA: produtos naturais e seus derivados. Drug Discov Today, 2(1), $204-207$.

Pavan, F. R., Leite, C. Q. F., Coelho, R. G., Coutinho, I. D., Honda, N. K., Cardoso, C. A. L., Vilegas, W., Leite, S. R. de A., Sato, D. N. (2009). Evaluation of anti Mycobacterium tuberculosis activity of Campomanesia adamantium (Myrtaceae), Química Nova 32 (5).

Prakash, B., Kumar. A., Prem Pratap Singh, \&Songachan, L.S. (2020). Antimicrobial andantioxidant properties of phytochemicals: Current status and future perspective,Editor(s): Bhanu Prakash, Functional and Preservative Properties of Phytochemicals. Academic Press, 1-45.

Quideau S., Deffieux D., Douat-Casassus C., Pouységu L. (2011). Polifenóis Vegetais: Propriedades Químicas, Atividades Biológicas e Sintese. Chem. Int. Ed. Engle, 17 (1), 586-621.

Rocha, A. R. F., Sousa, H. G., Vale Júnior, E. P., Lima, F. L., Costa, A. S. G., Araújo, A. R., Leite, J. R. S. A., Martins, F. A., Oliveira, M. B. P. P., Plácido, A., Filho, F. S. S., \& Lago, E. C. (2021). Extract sand fractions of Croton L. (Euphorbiaceae) species with antimicrobial activi tyandanti oxidant potential, $L W T, 139$ (1)

Rodríguez-Baño, J., Rossolini, G. M, Schultsz, C., Tacconelli, E., Murthy, S., Ohmagari, N., Holmes, A., Bachmann, T., Goossens, H., Canton, R., Roberts, A. P., Henriques-Normark, B., Clancy, C. J., Huttner, B., Fagerstedt, P., Lahiri, S., Kaushic, C., Hoffman, S. J., Warren, M., Zoubiane, G., Essack, S., Laxminarayan, R., Plant, L. (2021). Antimicrobial resistance research in a post-pandemic world: Insights on antimicrobial resistance research in the COVID19 pandemic. Journal of Global Antimicrobial Resistance, 25 (1), 5-7.

Sá, S., Chaul, L.T., Alves,V. F., Fiuza, T.S., Tresvenzol, L. M. F., Vaz, B. G., Ferri, P. H., Borges, L. L., \& Paula, J. R. (2018). Phytochemistry and antimicrobial activity of Campomanesiaadamantium, Revista Brasileira de Farmacognosia, Volume 28, Issue 3, Pages 303- 11.

Sandelowski M, Barroso J. Handbook for synthesizing qualitati ve research. Brainerd: Bang Printi ng; 2007.

Silva, D. H. S., Castro-Gamboa, I.,\&Bolzani, V. S. (2010). 3.05 Plant Diversity from Brazilian Cerrado and Atlantic Forest as a Tool for Prospecting Potential Therapeutic Drugs, Editor(s): Hung-Wen (Ben) Liu, Lew Mander, Comprehensive Natural Products II, Elsevier, 95-133.

Silva, S. C. C. C., Braz, E. M. A., Carvalho, F. A. A., Brito, C. A. R. S., Brito, L. M., Barreto, H. M., Filho, E. C. S., \& Silva, D. A. (2020) Antibacterial and cytotoxic properties from esterified Sterculia gum, International Journal of Biological Macromolecules, 164 (1), $606-615$. 
Simonetti, E.; Ethur, M.E.; Castro, L.C.; Kauffmann, C.; Giacomin, A.C.; Ledur, A.; Arossi, K.; Pacheco, L.A.; Goettert, M.I.; Faleiro, D.; Freitas, E.M. (2016) Avaliação da atividade antimicrobiana de extratos de Eugenia anomala e Psidium salutare (Myrtaceae) frente à Escherichia coli e Listeria monocytogenes. Rev. Bras. Pl. Med., 18 (1), 9-18.

Sobral, M., Proença, C., Souza, M., Mazine, F., Lucas, E. (2015). Myrtaceae in Lista de Espécies da Flora do Brasil. Jardim Botânico do Rio de Janeiro. Disponível em http://floradobrasil.jbrj.gov.br/jabot/floradobrasil/ FB171.

Stefanello M.É., Cervi A.C., Ito I.Y., Salvador M.J., Wisniewski Jr A., Simionatto E.L. (2008). Chemical composition and antimicrobial activity of essential oils of Eugenia chlorophylla (Myrtaceae). Journal of Essential Oil Research, 20 (1), 75-8.

Stone Sá, Luíza T. Chaul a, Virgínia F. Alves b, Tatiana S. Fiuzac, Leonice M.F. Tresvenzol a, Boniek G. Vaz d, Pedro H. Ferri d, Leonardo L. Borges e,f , José R. Paula. (2018). Phytochemistry and antimicrobial activity of Campomanesia adamantium. Revista Brasileira de Farmacognosia, 28 (1), 303-311

Tavares, T. D., Antunes, J. C., Padrão, J., Ribeiro, A. I., Zille, A. , Amorim, M. T. P., Ferreira, F., Felgueiras, H. P. (2020). Activity Of Specialized Biomolecules Against Gram-Positive And Gram-Negative Bacteria. Antibiotics, 9 (6), 314 - 321.

Teixeira, N. et al. (2019). Edible fruits from Brazilian biodiversity: A review on their sensorial characteristics versus bioactivity as tool to select research. Food research international, 119 (1), 325-348.

Vizzotto, M., Krolow, A. C. R., \& Weber, G. E. B. (2010). Metabólitos secundários encontrados em plantas e sua importância. Embrapa Clima TemperadoDocumento 316, 1 (1), 1-16. Disponível em https:/www.infoteca.cnptia.embrapa.br/bitstream/doc/886074/1/documento316.pdf

Waters, V. J.,Kidd, T. J., Canton, R., Ekkelenkamp, M. B., Johansen, H. K., LiPuma, J. J., Bell, S. C., Elborn, J. S., Flume, P. A., VanDevanter, D. R., \&Gilligan, P. (2019). Antimicrobial Resistance International Working Group in Cystic Fibrosis. Reconciling Antimicrobial Susceptibility Testingand Clinical Response in Antimicrobial Treatment of Chronic Cystic Fibrosis LungInfections. Clinical Infectious Diseases, 69 (10).

Zacchino SA, Butassi E., Liberto MD, Raimondi M., Postigo A., Sortino M. (2017). Plant phenolics and terpenoids as adjuvants of antibacterial and antifungal drug. Fitomedicina, 15 (1), 27-48. 